\title{
On a conjecture of Pappas and Rapoport about the standard local model for $\mathrm{GL}_{d}$
}

By Dinakar Muthiah at Kashiwa, Alex Weekes at Vancouver and Oded Yacobi at Sydney

\begin{abstract}
In their study of local models of Shimura varieties for totally ramified extensions, Pappas and Rapoport posed a conjecture about the reducedness of a certain subscheme of $n \times n$ matrices. We give a positive answer to their conjecture in full generality. Our main ideas follow naturally from two of our previous works. The first is our proof of a conjecture of Kreiman, Lakshmibai, Magyar, and Weyman on the equations defining type A affine Grassmannians. The second is the work of the first two authors and Kamnitzer on affine Grassmannian slices and their reduced scheme structure. We also present a version of our argument that is almost completely elementary: the only non-elementary ingredient is the Frobenius splitting of Schubert varieties.
\end{abstract}

\section{Introduction}

For integers $n, e \geq 2$, Pappas and Rapoport conjectured that the following subscheme:

$$
\mathcal{N}_{n, e}=\left\{A \in \operatorname{Mat}_{n \times n}: A^{e}=0, \operatorname{det}(\lambda-A)=\lambda^{n}\right\}
$$

of $n \times n$ matrices is reduced [11, Conjecture 5.8] (see also [12, Section 6]). Our main result is a proof of this conjecture (Theorem 3.6).

Soon after the Pappas-Rapoport conjecture was announced in 2000, Weyman ([15]) proved two cases: (i) when the base field is characteristic zero, and (ii) when the base field has arbitrary characteristic and $e=2$. Our main contribution is proving the conjecture in positive characteristic, which is the interesting case for arithmetic applications. We make no assumptions on the characteristic, so our argument also gives a new proof in characteristic zero.

The corresponding author is Oded Yacobi.

Dinakar Muthiah was supported by JSPS KAKENHI Grant Number JP19K14495. Alex Weekes was supported in part by the Perimeter Institute for Theoretical Physics. Research at Perimeter Institute is supported by the Government of Canada through Innovation, Science and Economic Development Canada and by the Province of Ontario through the Ministry of Research, Innovation and Science. Oded Yacobi is supported by the ARC grant DP180102563.

(๕)BY (C) 2020 Dinakar Muthiah, Alex Weekes and Oded Yacobi, published by De Gruyter. This work is licensed under the Creative Commons Attribution 4.0 International License. 
Pappas and Rapoport also proved interesting results about nilpotent orbits and affine Grassmannians conditional upon the reducedness of $\mathcal{N}_{n, e}$ (see [11, equation (5.26), Proposition 6.5, Proposition 6.6]). We can now state these results unconditionally.

Pappas and Rapoport's conjecture arose in their investigation of local models of Shimura varieties. Shimura varieties serve as a bridge between arithmetic geometry and automorphic forms, and as such they play an important role in the Langlands program. Local models of Shimura varieties, which capture the behavior that occurs when considering reduction mod $p$, often allow one to reduce arithmetic problems to questions about affine Grassmannians and flag varieties ([13]). These problems tend to be difficult but often tractible (e.g. $[5,6,16])$.

1.1. Our approach. Our solution follows naturally by building on the ideas of our previous work [10] and earlier work by the first two authors and Kamnitzer [7]. One of our stated goals in [10, Section 1.3.2] is to use the framework developed there to understand the scheme structure of nilpotent orbit closures in positive characteristic. The present work is our first success in this program.

In [10], we proved a conjecture of Kreiman, Lakshmibai, Magyar, and Weyman about the equations defining affine Grassmannians for $\mathrm{SL}_{n}$. Their conjecture is that the defining equations are given by certain explicit shuffle operators.

Our study led us to consider the general situation of a nilpotent operator $T$ on a vector space $V$. We consider the Grassmannian $\operatorname{Gr}(n, V)$, and define a subscheme $\delta^{T}$ by explicit $T$-shuffle operators generalizing the shuffle operators (cf. Section 2.1).

1.1.1. An alternate definition of $\boldsymbol{s}^{\boldsymbol{T}}$. Let $\boldsymbol{\delta}^{T}$ be the subscheme of the Grassmannian $\operatorname{Gr}(n, V)$ consisting of $T$-invariant $n$-planes $U$ such that $\operatorname{det}\left(\lambda-\left.T\right|_{U}\right)=\lambda^{n}$. Our first result (Theorem 2.3) is that ${ }^{\prime} \delta^{T}=\delta^{T}$. Theorem 2.3 should be of general interest because ${ }^{\prime} \delta^{T}$ is given as a moduli functor, while $\delta^{T}$ is given by explicit homogeneous equations in the Plücker embedding of the Grassmannian.

More can be said in the special case where $\operatorname{dim} V=d e$ for positive integers $d, e$, and $T$ has Jordan type given by the partition $\left(e^{d}\right)$. Let ${ }^{T} \delta^{T}$ denote the preimage of ${ }^{\prime} \delta^{T}$ in the Stiefel variety. Then we have natural maps:

$$
s^{T} \stackrel{\pi}{\longleftarrow} \widetilde{\delta^{T}} \stackrel{\varphi}{\longrightarrow} \mathcal{N}_{n, e} .
$$

See Section 2.1 and Section 2.3 for explanation. The key fact is the following.

Theorem 1.1. The morphism $\widetilde{\AA^{T}} \stackrel{\varphi}{\longrightarrow} \mathcal{N}_{n, e}$ is smooth.

This is a corollary of one of Pappas and Rapoport's main theorems [11, Theorem 4.1]. For the benefit of the reader we explain in Section 2.3 how their proof works in our simpler setting.

Pappas and Rapoport consider a version of (1.1) over a discrete valuation ring that base changes to (1.1) over the residue field. In particular, the object that base changes to ${ }^{\prime} \delta^{T}$ is a particular local model. In this article, we prove that ${ }^{\prime} \delta^{T}$ is reduced, which implies that this local model is flat over its discrete valuation ring of definition.

1.1.2. Applying our previous results and Frobenius splitting. In [7], we developed some techniques for attacking a conjecture about the reduced scheme structure on Schubert 
varieties in the affine Grassmannian. We use this to prove that a certain family of $\delta^{T}$ are reduced (Proposition 3.1). In particular, we show that these schemes are Schubert varieties in the affine Grassmannian with their reduced scheme structure. Using the fact that Schubert varieties are Frobenius split compatibly with their Schubert subvarieties, we can take intersections and conclude that a larger family of $\widetilde{\delta^{T}}$ are also reduced (Proposition 3.3). It follows that all corresponding $\widetilde{\delta^{T}}$ are reduced, since $\widetilde{\delta^{T}} \rightarrow \delta^{T}$ is a $\mathrm{GL}_{n}$-torsor.

We note that this strategy is similar to the proof of [4, Theorem 4.5.1], where the special fiber $\bar{M}^{\text {loc }}$ of a local model is expressed as an intersection of smooth Schubert varieties. One additional difficulty for us is that we consider intersections of singular Schubert varieties.

Finally, we show that for each $n$ and $e$ there is some ${ }^{\prime} \delta^{T}$ (which we have shown is reduced) such that the corresponding map $\widehat{\delta^{T}} \rightarrow \mathcal{N}_{n, e}$ is surjective. Surjectivity is a direct calculation (Proposition 3.5). We also know that this map is smooth, and reducedness descends along smooth surjections. Therefore, we conclude that $\mathcal{N}_{n, e}$ is reduced.

After analyzing our argument, we found a simplified but less conceptual version that is almost completely elementary. The only non-elementary ingredient is the Frobenius splitting of Schubert varieties. We present this simplified argument in Section 3.1.

Acknowledgement. We would like to thank an anonymous referee for their helpful report, and also the referee of [10] for suggesting the connection between our work and that of Pappas and Rapoport. We thank Geordie Williamson and Xinwen Zhu for their comments on an earlier version of this manuscript. We are also grateful to the Centre de Recherches Mathématiques and to Joel Kamnitzer and Hugh Thomas for organizing the program "Quiver Varieties and Representation Theory" where this project was started.

\section{Preliminaries}

2.1. Grassmannians and associated schemes. Let $\mathbb{k}$ be a field of arbitrary characteristic. Because our goal is to prove reducedness of a scheme, we may assume $\mathbb{k}$ is algebraically closed. Let $V$ be a finite-dimensional vector space over $\mathbb{k}$, and let $n$ be a non-negative integer. Write $\operatorname{Gr}(n, V)$ for the Grassmannian of $n$-planes in $V$. Let $\underline{V}$ denote the trivial bundle on $\operatorname{Gr}(n, V)$ attached to $V$, and let $\mathcal{T}$ denote the tautological rank- $n$ vector subbundle of $\underline{V}$. Write $\mathcal{O}(-1)=\bigwedge^{n} \mathcal{T}$, which is a line subbundle of the trivial bundle $\bigwedge^{n} V$. This defines the Plücker embedding $\operatorname{Gr}(n, V) \hookrightarrow \mathbb{P}\left(\bigwedge^{n} V\right)$.

Further, let us assume that $T: V \rightarrow V$ is a nilpotent operator. We denote by $\mathscr{g}^{T}(n, V)$ the subscheme of $\operatorname{Gr}(n, V)$ consisting of $T$-invariant subspaces (see e.g. [10, Section 5.4]). When $n$ and $V$ are clear from context, we will simply write $\mathcal{E}^{T}=\mathscr{E}^{T}(n, V)$.

For any $T$-invariant $n$-plane $U$, we consider the characteristic polynomial $\operatorname{det}\left(\lambda-\left.T\right|_{U}\right)$. This defines a regular function $\chi: \mathscr{E}^{T} \rightarrow \mathbb{k}[\lambda]$. Notice that set theoretically $\chi$ is equal to $\lambda^{n}$, but this is not necessarily true scheme-theoretically. In particular, the non-leading coefficients of $\chi$ are all nilpotent elements of $\mathbb{k}\left[\mathscr{E}^{T}\right]$. We define ${ }^{\prime} \delta^{T}={ }^{\prime} \delta^{T}(n, V)$ to be the closed subscheme of $\mathscr{G}^{T}$ defined by the equation $\chi=\lambda^{n}$.

We define for each $d \geq 1$ shuffle operators, which are linear operators

$$
\operatorname{sh}_{d}^{T}: \bigwedge^{n} V \rightarrow \bigwedge^{n} V
$$

To define these, consider the $\mathbb{k}[z]$-linear operator $I+z T$ on $V \otimes \mathbb{k}[z]$, and its $n$-th wedge 
power $\bigwedge^{n}(1+z T)$ (taken over $\mathbb{k}[z]$ ). The following equality of operators on $\bigwedge^{n}(V) \otimes \mathbb{k}[z]$ defines $\operatorname{sh}_{d}^{T}$ :

$$
\bigwedge^{n}(I+z T)=I+\sum_{d=1}^{n} z^{d} \operatorname{sh}_{d}^{T}
$$

These operators were introduced in [10, Section 6.1], where also an explicit formula appears.

For each $\mathrm{sh}_{d}^{T}$ we can consider the vanishing locus, denoted $\mathbb{V}\left(\mathrm{sh}_{d}^{T}\right)$, which is the subvariety of $\mathbb{P}\left(\bigwedge^{n} V\right)$ given by the projectivization of the kernel of $\operatorname{sh}_{d}^{T}$. We define

$$
s^{T}=s^{T}(n, V)=\bigcap_{d \geq 1} \mathbb{V}\left(\operatorname{sh}_{d}^{T}\right) \cap \operatorname{Gr}(n, V),
$$

and we refer to the homogeneous ideal defining $8^{T}$ as the shuffle ideal. In our previous work, we proved the following.

Theorem 2.1 ([10, Theorem 6.4 and Proposition 6.6]). The subscheme $8^{T}$ is a closed subscheme of $\mathscr{E}^{T}$. This closed embedding induces an isomorphism of reduced schemes.

Remark 2.2. In fact, we conjecture ([10, Conjecture 7.3]) that $\delta^{T}$ is reduced and is therefore equal to the reduced scheme of $e^{T}$.

The Stiefel variety $\overline{\operatorname{Gr}(n, V)}$ is the open subscheme of $\operatorname{Hom}_{\mathbb{k}}\left(\mathbb{k}^{n}, V\right)$ consisting of rank- $n$ linear transformations. We have a natural map $\overline{\operatorname{Gr}(n, V)} \rightarrow \operatorname{Gr}(n, V)$, which is a $\mathrm{GL}_{n}$-torsor. For any closed subscheme $X \hookrightarrow \operatorname{Gr}(n, V)$, we define $\widetilde{X}$ to be the preimage of $X$ inside the Stiefel variety. For example, we define the scheme $\widetilde{\delta^{T}}$ this way.

\subsection{Alternative description of $\boldsymbol{s}^{T}$.}

Theorem 2.3. We have an equality $\varsigma^{T}=' \AA^{T}$ as subschemes of $\operatorname{Gr}(n, V)$.

Proof. Recall that $8^{T}$ is defined by intersecting the Grassmannian $\operatorname{Gr}(n, V)$ with the vanishing locus of shuffle operators $\operatorname{sh}_{d}^{T}: \bigwedge^{n} V \rightarrow \bigwedge^{n} V$. Consider these as operators on the

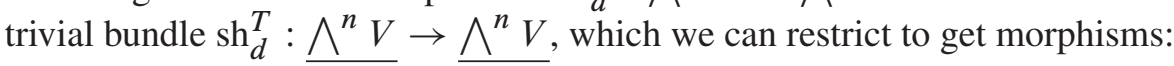

$$
\operatorname{sh}_{d}^{T}: \mathcal{O}(-1) \rightarrow \bigwedge^{n} V .
$$

One can alternately define $\delta^{T}$ to be the intersection of the vanishing loci of (2.2). Observe by Theorem 2.1 that over $\mathcal{E}^{T}$, the maps

$$
\operatorname{sh}_{d}^{T}: \mathcal{O}(-1) \rightarrow \bigwedge^{n} V
$$

factor through maps $\operatorname{sh}_{d}^{T}: \mathcal{O}(-1) \rightarrow \mathcal{O}(-1)$.

Write $\mathbb{A}^{1}=\operatorname{Spec} \mathbb{k}[z]$. We can and will interpret (2.1) as an equality of endomorphisms of $\bigwedge^{n} V \otimes \mathbb{k}[z]$, the trivial bundle on $\operatorname{Gr}(n, V) \times \mathbb{A}^{1}$ with fiber $\bigwedge^{n} V$. If we restrict to $\mathscr{E}^{T} \times \mathbb{A}^{1}$, we obtain a map

$$
I+\sum_{d=1}^{n} z^{d} \operatorname{sh}_{d}^{T}: \mathcal{O}(-1) \otimes \mathbb{k}[z] \rightarrow \mathcal{O}(-1) \otimes \mathbb{k}[z]
$$


Because $\mathcal{O}(-1)$ is a line bundle, the map (2.3) is equivalent to the data of a regular function on $\mathscr{G}^{T}$ valued in $\mathbb{k}[z]$. By (2.1), this map is given by the $z^{n} \chi\left(-z^{-1}\right)$, where $\chi: \mathscr{G}^{T} \rightarrow \mathbb{k}[z]$ is the characteristic polynomial map defined above.

2.3. Smoothness of the map $\varphi: \widetilde{\boldsymbol{s}^{T}} \rightarrow \mathcal{N}_{n, e}$. We have a map $\varphi: \widetilde{\AA^{T}} \rightarrow \operatorname{Mat}_{n \times n}$ defined as follows. For a test ring $R$, we have

$$
\widetilde{\mathcal{s}^{T}}(R)=\left\{(U, \psi): U \in \mathcal{\prime}^{\prime}(R) \text { and } \psi: R^{n} \stackrel{\sim}{\rightarrow} U\right\} .
$$

The map $\varphi$ sends a pair $(U, \psi)$ to the $n \times n$ matrix $\psi^{-1} \circ T \circ \psi$.

Because of the characteristic polynomial equation defining ${ }^{\prime} \delta^{T}$, the image of $\varphi$ lies in the nilpotent cone $\mathcal{N}$ (even scheme theoretically). In general this map is poorly behaved, but in the special case we consider below it is smooth.

Let $d, e \geq 1$ be integers, let $V$ be a vector space of dimension $d e$ and suppose $T$ is the nilpotent endomorphism of $V$ that is given by a standard Jordan form corresponding to the partition $\left(e^{d}\right)$. Let ${ }^{\prime} \delta^{T}={ }^{\prime} \delta^{T}(n, V)$ for some $n \leq d e$. In this case, the morphism $\varphi$ factors through a map $\varphi: \AA^{T} \rightarrow \mathcal{N}_{n, e}$.

Theorem 2.4 ([11, Theorem 4.1]). The morphism $\varphi: \widetilde{\AA^{T}} \rightarrow \mathcal{N}_{n, e}$ is smooth of relative dimension $n d$.

The proof of [11, Theorem 4.1] holds in a more complicated arithmetic situation, and it simplifies greatly in the present one. For the benefit of the reader, we will briefly explain how their proof works in our situation.

Define a closed subscheme $\mathcal{V}$ of $\operatorname{Mat}_{n, n} \times \operatorname{Hom}_{\mathbb{k}}\left(\mathbb{k}^{n}, V\right)$ as follows. For any test ring $R$, consider the ring $S=R[t] / t^{e}$. The operator $T$ defines an $S$-module structure on $V \otimes R$. Additionally, any point $A \in \mathcal{N}_{n, e}(R)$ defines an $S$-module structure on $R^{n}$, which we denote by $R_{A}^{n}$. We define $\mathcal{V}$ by

$$
\mathcal{V}(R)=\left\{(A, \varphi): A \in \mathcal{N}_{n, e}(R) \text { and } \varphi \in \operatorname{Hom}_{S}\left(R_{A}^{n}, V \otimes R\right)\right\} .
$$

It is clear that $\widetilde{s^{T}}$ admits an open embedding into $\mathcal{V}$ with image equal to the set of pairs $(A, \varphi)$ where $\varphi$ is of maximal rank.

We also have a map $\mathcal{V} \rightarrow \mathcal{N}_{n, e}$, but for general $T$ this map will not be well behaved. However, our assumptions on the Jordan type of $T$ imply that $V \otimes R$ is a free $S$-module of rank $d$. Noting that there is an isomorphism

$$
\operatorname{Hom}_{R}\left(R^{n}, R\right) \stackrel{\sim}{\rightarrow} \operatorname{Hom}_{S}\left(R_{A}^{n}, S\right)
$$

given by $f \mapsto \varphi$, where $\varphi(v)=\sum_{i} f\left(A^{e-i-1} v\right) t^{i}$, one concludes that the map $\mathcal{V} \rightarrow \mathcal{N}_{n, e}$ is a (trivial) vector bundle of rank $n d$. Therefore, the map $\widehat{\mathcal{S}^{T}} \rightarrow \mathcal{N}_{n, e}$ is smooth because it factors as an open embedding into a vector bundle followed by projection from the vector bundle to its base.

Remark 2.5. For a general $T$ with $T^{e}=0$, the fibers of $\mathcal{V} \rightarrow \mathcal{N}_{n, e}$ are closely related to the work of Shayman [14, Section 5], who studied certain locally closed subvarieties of the varieties ${ }^{\prime}{ }^{T}$. 
2.4. Type $\boldsymbol{A}$ affine Grassmannians. Let $n \geq 2$. Recall the $\mathrm{GL}_{n}$ affine Grassmannian $\mathscr{G}_{\mathrm{GL}_{n}}$ parameterizing lattices $L \subset \mathbb{k}((t))^{n}$ such $t^{a} L_{0} \subseteq L \subseteq t^{-b} L_{0}$ for some integers $a, b \geq 0$ where $L_{0}=\mathbb{k}[[t]]^{n}$ (see e.g. [17, Section 1.1]). We further require that

$$
\operatorname{dim}_{\mathbb{k}}\left(L \cap L_{0}\right) / L_{0}-\operatorname{dim}_{\mathbb{k}}\left(L \cap L_{0}\right) / L=0 .
$$

This condition means that we are only considering the connected component of the full affine Grassmannian of $\mathrm{GL}_{n}$ containing the point $L_{0}$. We will not use the other connected components, so our notation should cause no confusion.

For each pair of integers $a, b \geq 0$, we consider the linear operator $T_{a, b}$ on $t^{-b} L_{0} / t^{a} L_{0}$ given by multiplication by $t$. We therefore obtain a closed embedding

$$
\mathscr{E}^{T_{a, b}}=\mathscr{E}^{T_{a, b}}\left(n a, t^{-b} L_{0} / t^{a} L_{0}\right) \hookrightarrow \mathcal{E}_{\mathrm{GL}_{n}} .
$$

Explicitly, we identify $\mathscr{E}^{T_{a, b}}$ with the subscheme of $\mathscr{G}_{\mathrm{GL}_{n}}$ parameterizing lattices $L \subset \mathbb{k}((t))^{n}$ such that $t^{a} L_{0} \subset L \subset t^{-b} L_{0}$ and $\operatorname{dim}\left(L / t^{a} L_{0}\right)=n a$. Taking direct limits, we have

$$
\mathcal{E}_{\mathrm{GL}_{n}}=\lim _{a, b \rightarrow \infty} e^{T_{a, b}} .
$$

This realizes $\mathcal{E}_{\mathrm{GL}_{n}}$ as an ind-scheme of ind-finite type. We define the affine Grassmannian $\mathscr{E}_{\mathrm{SL}_{n}}$ for $\mathrm{SL}_{n}$ to be the induced reduced structure of $\mathcal{G}_{\mathrm{GL}_{n}}$. Explicitly:

$$
\mathscr{E}_{\mathrm{SL}_{n}}=\lim _{a, b \rightarrow \infty}\left(\mathscr{E}^{T_{a, b}}\right)^{\mathrm{red}}
$$

We may also consider $8^{T_{a, b}}=8^{T_{a, b}}\left(n a, t^{-b} L_{0} / t^{a} L_{0}\right)$. A priori, $8^{T_{a, b}}$ is a subscheme of $\operatorname{Sr}_{\mathrm{GL}_{n}}$, but below (Corollary 3.7) we will show that $\delta^{T_{a, b}}$ is in fact equal to the reduced scheme $\left(\mathscr{G}^{T_{a, b}}\right)^{\mathrm{red}}$ and is therefore a subscheme of $\mathcal{E}_{\mathrm{SL}_{n}}$.

2.4.1. Big cells. Consider the decomposition

$$
\mathbb{k}((t))^{n}=L_{0} \oplus t^{-1} \mathbb{k}\left[t^{-1}\right]^{n}
$$

and the induced projection map $\mathbb{k}((t))^{n} \rightarrow L_{0}$. The big cell of $\mathscr{G}_{\mathrm{GL}_{n}}$ is the open locus consisting of lattices $L$ such that the restricted projection map $L \rightarrow L_{0}$ is an isomorphism. As is well known (see e.g. [3, Lemma 2]), the big cell is isomorphic to the ind-scheme $\mathrm{GL}_{n}^{(1)}\left[t^{-1}\right]$ whose $R$-points are given by $\left\{A\left(t^{-1}\right) \in \mathrm{GL}_{n}\left(R\left[t^{-1}\right]\right): A\left(t^{-1}\right) \equiv 1 \bmod t^{-1}\right\}$. The open immersion $\mathrm{GL}_{n}^{(1)}\left[t^{-1}\right] \hookrightarrow \mathcal{G r}_{\mathrm{GL}_{n}}$ is given by sending a matrix polynomial $A\left(t^{-1}\right)$ to the lattice spanned by its columns. We will use this identification and simply write $\mathrm{GL}_{n}^{(1)}\left[t^{-1}\right]$ for the big cell.

We can intersect the big cell of $\mathcal{G}_{\mathrm{GL}_{n}}$ with $\mathcal{E}_{\mathrm{SL}_{n}}$ to obtain the big cell of the latter. As above, we can identify the big cell of $\mathcal{E}_{\mathrm{SL}_{n}}$ with $\mathrm{SL}_{n}^{(1)}\left[t^{-1}\right]$, which is analogously defined.

Let $\mathrm{SL}_{n}[[t]]$ be the (infinite-type) scheme whose $R$-points are given by $\mathrm{SL}_{n}(R[[t]])$. Observe that the group $\mathrm{SL}_{n}[[t]]$ acts on $\mathcal{E}_{\mathrm{GL}_{n}}$ by left multiplication. Note that every orbit meets the big cell as we consider only the $L_{0}$ connected component of the full affine Grassmannian of $\mathrm{GL}_{n}$.

2.4.2. Schubert varieties. Recall that the $\mathrm{SL}_{n}[[t]]$ orbits on $\mathcal{G}_{\mathrm{SL}_{n}}$ are indexed by dominant cocharacters. For a dominant cocharacter $\mu$, let $\overline{\mathscr{E}_{\mathrm{r}}}{ }^{\mu}$ be the corresponding orbit closure in $\mathscr{G}_{\mathrm{SL}_{n}}$. By definition $\overline{\mathcal{E}_{\mathrm{r}}^{\mu}}$ has reduced scheme structure. Additionally, $\mathscr{E}_{\mathrm{SL}_{n}}$ is isomorphic 
to a partial flag variety for the Kac-Moody group $\widehat{\mathrm{SL}_{n}}$, and the subvarieties $\overline{\mathcal{E}_{\mathrm{r}}^{\mu}}$ are Schubert subvarieties. In particular, each $\overline{\mathscr{E}_{\mathrm{r}}}{ }$ admits a Frobenius splitting compatible with all of its Schubert subvarieties ([9, Chapter VIII], see also [3, Section 4] and [2, Chapter 2]). Therefore, the scheme theoretic intersection of two Schubert varieties is Frobenius split and hence reduced. This result is the only consequence of Frobenius splitting we will use.

The Schubert varieties relevant to us are $\overline{\mathcal{E}_{\mathrm{r}} p n \varpi_{1}^{\vee}}$ and $\overline{\mathcal{E r}_{\mathrm{r}} q n \varpi_{n-1}^{\vee}}$, where $p, q \geq 1$ are integers. Here $n \varpi_{1}^{\vee}$ and $n \varpi_{n-1}^{\vee}$ are $n$ times the first and last fundamental coweight, which are the minimal multiples that lie in the cocharacter (equivalently, coroot) lattice for SL $\mathrm{S}_{n}$. Recall the standard identification of the cocharacter lattice of $\mathrm{SL}_{n}$ with elements of $\mathbb{Z}^{n}$ whose entries sum to zero. Under this identification we have

$$
n \varpi_{1}=(n-1,-1, \ldots,-1) \text { and } n \varpi_{n-1}=(1, \ldots, 1,1-n) .
$$

We have

$$
\overline{\mathscr{E}_{\mathrm{r}} p n \varpi_{1}^{\vee}}=\left(\mathscr{E}^{T_{p(n-1), p}}\right)^{\mathrm{red}}, \quad \overline{\mathscr{E}_{\mathrm{r}} q n \varpi_{n-1}^{\vee}}=\left(\mathscr{E}^{T_{q, q(n-1)}}\right)^{\mathrm{red}},
$$

which is an explicit set-theoretic description of these Schubert varieties in terms of lattices. Because of Theorem 2.1, one immediately obtains closed embeddings

$$
\overline{\mathscr{E}_{\mathrm{r}} p n \varpi_{1}^{\vee}} \hookrightarrow \delta^{T_{p(n-1), p}}, \quad \overline{\mathscr{E}_{\mathrm{r}} q n \varpi_{n-1}^{\vee}} \hookrightarrow \delta^{T_{q, q(n-1)}}
$$

that are bijective on points. Below (Proposition 3.1) we will show that these are actually isomorphisms of schemes. In particular, the naïve lattice description of these Schubert varieties is scheme-theoretically correct once we add a further condition about the characteristic polynomial of $t$ (as in the definition of ${ }^{\prime} \delta^{T}$ ).

2.4.3. Diagram automorphism. Presentation (2.4) gives rise to a closed embedding of $\operatorname{Sr}_{\mathrm{GL}_{n}}$ into the Sato Grassmannian $\mathrm{SGr}=\lim _{a, b \rightarrow \infty} \operatorname{Gr}\left(n a, t^{-b} L_{0} / t^{a} L_{0}\right)$. Taking direct limits of the Plücker embeddings gives the Plücker embedding of $\operatorname{SGr}$ into $\mathbb{P}(\mathcal{F})$, where $\mathcal{F}$ is the Fermion Fock space. As is well known (see for example [10, Section 4.1]), the Fermion Fock space can be identified with the vector space underlying the ring of symmetric functions.

Consider the involution of the ring of symmetric functions given by swapping elementary and homogeneous symmetric functions (denoted $\omega$ in [8, Section I.2]). This induces an involution $\omega$ of SGr that preserves $\mathcal{E}_{\mathrm{GL}_{n}}$ and $\mathscr{E}_{\mathrm{S}_{\mathrm{S}_{n}}}$. On the level of Schubert varieties, $\omega$ maps $\overline{\mathscr{E}_{\mathrm{r}} \mu}$ isomorphically onto $\overline{\mathscr{E}_{\mathrm{r}} \mu^{*}}$, where $*$ is induced by the unique non-trivial diagram automorphism of $\mathrm{SL}_{n}$. In particular, $\omega$ maps $\overline{\mathscr{E}_{\mathrm{r}} p n \varpi_{1}^{\vee}}$ isomorphically onto $\overline{\mathscr{E}_{\mathrm{r}} p n \varpi_{n-1}^{\vee}}$. On the level of big cells $\mathrm{GL}_{n}^{(1)}\left[t^{-1}\right]$ and $\mathrm{SL}_{n}^{(1)}\left[t^{-1}\right], \omega$ is given by the following map on matrix polynomials:

$$
A\left(t^{-1}\right) \mapsto J \cdot A\left((-1)^{n} t^{-1}\right)^{-T} \cdot J^{-1},
$$

where $J$ is the $n \times n$ antidiagonal matrix with entry $(-1)^{j}$ in column $j$, and where the superscript $-T$ denotes the inverse transpose.

Remark 2.6. Consider the group functor $\mathrm{SL}_{n}((t))$ whose $R$-points are $\mathrm{SL}_{n}(R((t)))$. It is common to think of the affine Grassmannian as the quotient $\mathrm{SL}_{n}((t)) / \mathrm{SL}_{n}[[t]]$. Equation (2.6) defines an involution of $\mathrm{SL}_{n}((t))$ which preserves $\mathrm{SL}_{n}[[t]]$, and hence induces an involution on $\mathrm{SL}_{n}((t)) / \mathrm{SL}_{n}[[t]]$. 
Note that there are subtle technicalities involved in precisely constructing the quotient $\mathrm{SL}_{n}((t)) / \mathrm{SL}_{n}[[t]]$, so for simplicity we have opted to define the affine Grassmannian via lattices. Therefore we have defined the involution via the embedding into $\mathbb{P}(\widetilde{F})$. However, the reader can safely consider the definition descending from $\mathrm{SL}_{n}((t))$.

\section{The main proof}

Proposition 3.1. For all $p, q \geq 1$, the embeddings

$$
\overline{\mathscr{G}_{\mathrm{r}} p n \varpi_{1}^{\vee}} \hookrightarrow \AA^{T_{p(n-1), p}} \quad \text { and } \overline{\mathscr{E}_{\mathrm{r}} q n \varpi_{n-1}^{\vee}} \hookrightarrow \varsigma^{T_{q, q(n-1)}}
$$

from (2.5) are isomorphisms. In particular, $\wp^{T_{p(n-1), p}}$ and $\wp^{T_{q, q(n-1)}}$ are reduced.

Proof. First consider the embedding $\overline{\mathcal{E}_{\mathrm{r}} p n \varpi_{1}^{\vee}} \hookrightarrow \delta^{T_{p(n-1), p}}$. Both spaces are invariant under the action of $\mathrm{SL}_{n}[[t]]$, so it suffices to check that this map is an isomorphism on the big cell GL ${ }_{n}^{(1)}\left[t^{-1}\right] \subseteq \operatorname{Gr}_{G L_{n}}$. Observe that the intersection $8^{T_{p(n-1), p}} \cap \mathrm{GL}_{n}^{(1)}\left[t^{-1}\right]$ maps into the closed subfunctor of $\mathrm{GL}_{n}^{(1)}\left[t^{-1}\right]$ consisting of matrix polynomials with degree less than or equal to $p$. In [7, Corollary 2] (see also the proof of [1, Proposition 6.1]), we proved that the intersection $\overline{\mathscr{S}_{\mathrm{r}} p n \varpi_{1}^{\vee}} \cap \mathrm{GL}_{n}^{(1)}\left[t^{-1}\right]$ is equal as a scheme to the subscheme of matrix polynomials

$$
A\left(t^{-1}\right)=1+A_{1} t^{-1}+\cdots+A_{p} t^{-p}
$$

such that $\operatorname{det}\left(A\left(t^{-1}\right)\right)=1$. In [10, Theorem 4.6] we showed that the coefficients of $\operatorname{det}\left(A\left(t^{-1}\right)\right)$ lie in the shuffle ideal defining $8^{T_{p(n-1), p}}$. Therefore the embedding is an isomorphism.

The second isomorphism follows by applying the diagram automorphism (Section 2.4.3) and observing that the shuffle ideal considered in [10] is invariant under it (see [10, Corollary 4.3, Theorem 4.6]).

Remark 3.2. Let $L$ be an $R$-point of $\oiint^{T_{p(n-1), p}} \cap \mathrm{GL}_{n}^{(1)}\left[t^{-1}\right]$. Then $L$ may be uniquely represented by a matrix polynomial $A\left(t^{-1}\right) \in \mathrm{GL}_{n}^{(1)}\left[t^{-1}\right]$. It is not difficult to show that there is an $R$-basis of $t^{-p} L_{0} / L$ under which the matrix of $t$ is the companion matrix of the matrix polynomial

$$
A\left(t^{-1}\right)=1+A_{1} t^{-1}+\cdots+A_{p} t^{-p} .
$$

In particular, the characteristic polynomial of this matrix is $\operatorname{det}\left(\lambda^{p n} \cdot A\left(\lambda^{-1}\right)\right)$. We see that the characteristic polynomial equation defining $\delta^{T_{p(n-1), p}}$ (via Theorem 2.3) corresponds exactly to the equation $\operatorname{det}\left(A\left(t^{-1}\right)\right)=1$ defining $\overline{E_{\mathrm{r}} p n \varpi_{1}^{\vee}} \cap \mathrm{GL}_{n}^{(1)}\left[t^{-1}\right]$ inside matrix polynomials with degree less than or equal to $p$. This in particular gives another proof of [10, Theorem 4.6].

Proposition 3.3. Let $p, q \geq 1$, and let $M=\min \{q, p(n-1)\}$ and $N=\min \{p, q(n-1)\}$. Then $\AA^{T_{M, N}}$ is reduced.

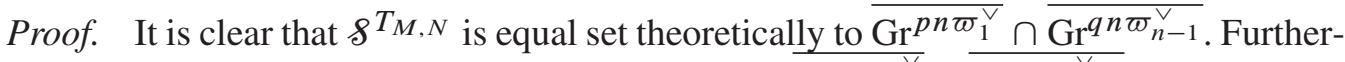

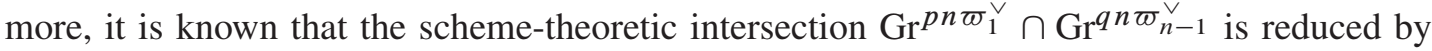
Frobenius splitting (see Section 2.4.2). We therefore obtain a closed embedding

$$
\S^{T_{M, N}} \hookrightarrow \delta^{T_{p(n-1), p}} \cap \wp^{T_{q, q(n-1)}}=\overline{\mathrm{Gr}^{p n \varpi_{1}^{\vee}}} \cap \overline{\mathrm{Gr}^{q n \varpi_{n-1}^{\vee}}}
$$

that is bijective on points. Because the target of this map is reduced, so is its source. 
Remark 3.4. The intersection $\overline{\mathscr{E}_{\mathrm{r}} p n \varpi_{1}^{\vee}} \cap \overline{\mathscr{G}_{\mathrm{r}} q n \varpi_{n-1}^{\vee}}$ is irreducible and is therefore also a Schubert variety for $\mathcal{E}_{\mathrm{SL}_{n}}$ (see [7, Proposition 5.4]). In fact, it is also not hard to see that $8^{T_{q, p}}$ is isomorphic to $\overline{\mathscr{E}_{\mathrm{r}} p n \varpi_{1}^{\vee}} \cap \overline{\mathscr{E}_{\mathrm{r}} q n \varpi_{n-1}^{\vee}}$ and is in particular reduced (cf. Corollary 3.7 below).

Recall by Theorem 2.3 and Theorem 2.4, we have a smooth map $\varphi: \widetilde{{\AA^{T_{1, e-1}}}^{\prime}} \rightarrow \mathcal{N}_{n, e}$.

Proposition 3.5. Let $e$ be an integer with $n \geq e \geq 1$. The map $\varphi: \overline{8^{T_{1, e-1}}} \rightarrow \mathcal{N}_{n, e}$ is surjective.

Proof. As surjectivity is a set-theoretic statement, it is sufficient to work with reduced schemes. Dividing with remainder, write $n=c e+f$, where $0 \leq f<e$, and let $\tau=\left(e^{c}, f\right)$ be the partition with $c$ parts of size $e$ and one part of size $f$. Then the reduced scheme of $\mathcal{N}_{n, e}$ is exactly the nilpotent orbit closure $\overline{\mathbb{O}_{\tau}} \subseteq$ Mat $_{n \times n}$. The map $\varphi$ is $\mathrm{GL}_{n}$-equivariant, so it suffices to check that every nilpotent orbit $\mathbb{O}_{\sigma} \subset \overline{\mathbb{O}_{\tau}}$ has non-empty intersection with the image. Since $\varphi$ is open (Theorem 2.4), it suffices to check that the zero orbit is in its image, and this is clear since $t$ acts trivially on $L_{0} / t L_{0}$.

Theorem 3.6. For every $n$, $e$ with $1 \leq e \leq n$, the scheme $\mathcal{N}_{n, e}$ is reduced.

Proof. The scheme $8^{T_{1, e-1}}$ is reduced by Proposition 3.3 (choosing $p=e-1$ and $q=1)$. The map

$$
\widetilde{8^{T_{1, e-1}}} \rightarrow 8^{T_{1, e-1}}
$$

is a torsor for $\mathrm{GL}_{n}$, so $\widetilde{\AA^{T_{1, e-1}}}$ is also reduced. By Theorem 2.4 and Proposition 3.5, the map

$$
\varphi: \widetilde{\AA^{T_{1, e-1}}} \rightarrow \mathcal{N}_{n, e}
$$

is smooth and surjective. In particular, it is faithfully flat, and the property of being reduced descends along faithfully flat morphisms.

3.1. Another proof. After closely inspecting the above argument, we found the following direct proof of Theorem 3.6. The idea is essentially the same as the more conceptual proof above, but gets to the punchline quickly and by more elementary means. The only nonelementary ingredient is the Frobenius splitting of Schubert varieties.

Another proof of Theorem 3.6. For any $p \geq 0$, define $Z_{p}$ to be the closed subscheme of Mat $_{n \times n}\left[t^{-1}\right]$ that represents the functor sending any test ring $R$ to:

$Z_{p}(R)=\left\{1+B_{1} t^{-1}+\cdots+B_{p} t^{-p} \in \operatorname{Mat}_{n \times n}\left(R\left[t^{-1}\right]\right): \operatorname{det}\left(1+B_{1} t^{-1} \cdots+B_{p} t^{p}\right)=1\right\}$.

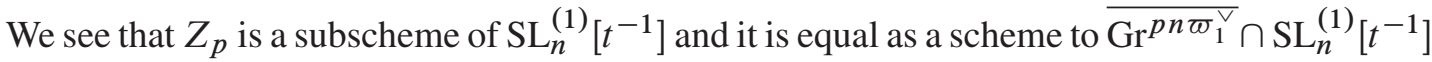
by [7, Corollary 2] (see also the proof of [1, Proposition 6.1]). In particular, $Z_{p}$ is reduced.

Let $X$ be the closed subscheme of Mat $_{n \times n}\left[t^{-1}\right]$ that represents the functor

$X(R)=\left\{1+C_{1} t^{-1}+\cdots+C_{n-1} t^{-(n-1)} \in \operatorname{Mat}_{n \times n}\left(R\left[t^{-1}\right]\right): \operatorname{det}\left(1-C_{1} t^{-1}\right)=1, C_{i}=C_{1}^{i}\right\}$.

Observe that the condition $\operatorname{det}\left(1-C_{1} t^{-1}\right)=1$ is equivalent to requiring that the characteristic 
polynomial of $C_{1}$ is $\lambda^{n}$. By the Cayley-Hamilton theorem, we have $C_{1}^{n}=0$. Therefore,

$$
1+C_{1} t^{-1}+\cdots+C_{n-1} t^{-(n-1)}=\left(1-C_{1} t^{-1}\right)^{-1}
$$

and

$$
\operatorname{det}\left(1+C_{1} t^{-1}+\cdots+C_{n-1} t^{-(n-1)}\right)=1 .
$$

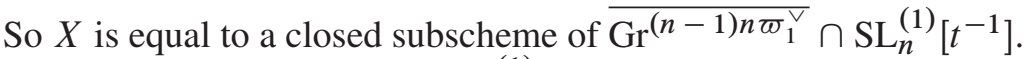

The involution (2.6) of $\mathrm{SL}_{n}^{(1)}\left[t^{-1}\right]$ gives an isomorphism between $X$ and $Z_{1}$. We there-

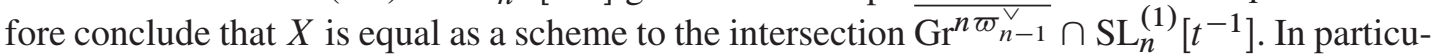
lar, $X$ is reduced. More directly, the map $X \rightarrow$ Mat $_{n \times n}$ sending $1+C_{1} t^{-1}+\cdots+C_{n-1} t^{-(n-1)}$ to $C_{1}$ induces an isomorphism between $X$ and the nilpotent cone $\mathcal{N}$.

Now consider the scheme theoretic intersection $X \cap Z_{e-1}$, which we compute inside $Z_{n-1}$. Because $Z_{n-1}$ admits a Frobenius splitting compatible with $X$ and $Z_{e-1}$, the intersection $X \cap Z_{e-1}$ is reduced.

On the other hand, we directly compute $\left(X \cap Z_{e-1}\right)(R)$ to be the set of

$$
1+C_{1} t^{-1}+\cdots+C_{n-1} t^{-(n-1)} \in \operatorname{Mat}_{n \times n}\left(R\left[t^{-1}\right]\right)
$$

such that $\operatorname{det}\left(1-C_{1} t^{-1}\right)=1, C_{i}=C_{1}^{i}$, and $C_{i}=0$ for $i \geq e$. Therefore, under the isomorphism $X \rightarrow \mathcal{N}$, we see that $X \cap Z_{e-1}$ maps isomorphically to $\mathcal{N}_{n, e}$.

Recall that all schemes of the form $\delta^{T_{a, b}}$ are reduced (Proposition 3.3, Remark 3.4). We can also argue this directly using the proof above. For all $a, b$, the map $\varphi: 8^{T_{a, b}} \rightarrow \mathcal{N}_{n a, a+b}$ is smooth (but not necessarily surjective). Because reducedness ascends along smooth maps, and $\delta^{T_{a, b}} \rightarrow \delta^{T_{a, b}}$ is a torsor for GL $\mathrm{GL}_{n}$, we obtain another proof of the following:

Corollary 3.7. For all $a, b \geq 1$, the scheme $8^{T_{a, b}}$ is reduced.

\section{References}

[1] A. Beauville and Y. Laszlo, Conformal blocks and generalized theta functions, Comm. Math. Phys. 164 (1994), no. 2, 385-419.

[2] M. Brion and S. Kumar, Frobenius splitting methods in geometry and representation theory, Progr. Math. 231, Birkhäuser, Boston 2005.

[3] G. Faltings, Algebraic loop groups and moduli spaces of bundles, J. Eur. Math. Soc. (JEMS) 5 (2003), no. 1, 41-68.

[4] U. Görtz, On the flatness of models of certain Shimura varieties of PEL-type, Math. Ann. 321 (2001), no. 3, 689-727.

[5] T. J. Haines and T. Richarz, Normality and Cohen-Macaulayness of parahoric local models, preprint 2019, https://arxiv.org/abs/1903.10585.

[6] X. He, Normality and Cohen-Macaulayness of local models of Shimura varieties, Duke Math. J. 162 (2013), no. $13,2509-2523$.

[7] J. Kamnitzer, D. Muthiah and A. Weekes, On a reducedness conjecture for spherical Schubert varieties and slices in the affine Grassmannian, Transform. Groups 23 (2018), no. 3, 707-722.

[8] I. G. Macdonald, Symmetric functions and Hall polynomials, Oxford Math. Monogr., The Clarendon Press, New York 1979.

[9] O. Mathieu, Formules de caractères pour les algèbres de Kac-Moody générales, Astérisque (1988), no. 159-160, 267.

[10] D. Muthiah, A. Weekes and $O$. Yacobi, The equations defining affine Grassmannians in type A and a conjecture of Kreiman, Lakshmibai, Magyar, and Weyman, Int. Math. Res. Not. (IMRN) 2020 (2020), Article ID rnaa131. 
[11] G. Pappas and M. Rapoport, Local models in the ramified case. I. The EL-case, J. Algebraic Geom. 12 (2003), no. $1,107-145$.

[12] G. Pappas, M. Rapoport and B. Smithling, Local models of Shimura varieties, I. Geometry and combinatorics, in: Handbook of moduli. Vol. III, Adv. Lect. Math. (ALM) 26, International Press, Somerville (2013), 135 217.

[13] M. Rapoport and T. Zink, Period spaces for $p$-divisible groups, Ann. of Math. Stud. 141, Princeton University Press, Princeton 1996.

[14] M. A. Shayman, On the variety of invariant subspaces of a finite-dimensional linear operator, Trans. Amer. Math. Soc. 274 (1982), no. 2, 721-747.

[15] J. Weyman, Two results on equations of nilpotent orbits, J. Algebraic Geom. 11 (2002), no. 4, 791-800.

[16] X. Zhu, On the coherence conjecture of Pappas and Rapoport, Ann. of Math. (2) 180 (2014), no. 1, 1-85.

[17] $X$. Zhu, An introduction to affine Grassmannians and the geometric Satake equivalence, in: Geometry of moduli spaces and representation theory, IAS/Park City Math. Ser. 24, American Mathematical Society, Providence (2017), 59-154.

Dinakar Muthiah, Kavli Institute for the Physics and Mathematics of the Universe (WPI), The University of Tokyo Institutes for Advanced Study, The University of Tokyo,

Kashiwa, Chiba 277-8583, Japan

e-mail: dinakar.muthiah@ipmu.jp

Alex Weekes, Department of Mathematics, University of British Columbia, 1984 Mathematics Road, Vancouver, BC V6T 1Z2, Canada e-mail: weekesal@math.ubc.ca

Oded Yacobi, School of Mathematics and Statistics, University of Sydney, Sydney, Australia e-mail: oded.yacobi@sydney.edu.au

Eingegangen 12. März 2020, in revidierter Fassung 6. August 2020 